\title{
EVALUATION OF A CHEMICAL RISK ASSESSMENT METHOD OF SOUTH KOREA FOR CHEMICALS CLASSIFIED AS CARCINOGENIC, MUTAGENIC OR REPROTOXIC (CMR)
}

\author{
MIN-UK KIM and SANG-HOON BYEON
}

\author{
Korea University, Seoul, South Korea \\ Department of Health Science
}

\begin{abstract}
Objectives: Chemicals were used in various fields by the development of industry and science and technology. The Chemical Hazard Risk Management (CHARM) was developed to assess the risk of chemicals in South Korea. In this study, we were to evaluate the CHARM model developed for the effective management of workplace chemicals. Material and Methods: We used 59 carcinogenic, mutagenic or reprotoxic (CMR) materials, which are both the work environment measurement result and the usage information among the manufacturer data. The CHARM model determines the risk to human health using the exposure level (based on working environment measurements or a combination of the quantity used and chemical physical properties (e.g., fugacity and volatility)), hazard (using occupational exposure limit (OEL) or Risk phrases (R-phrases)/Hazard statements (H-statements) from the Material Safety Data Sheet (MSDS)). Results: The risk level was lower when using the results of the work environment measurement than when applying the chemical quantity and physical properties in the exposure level evaluation method. It was evaluated as grade 4 for the CMR material in the hazard class determination. The risk assessment method by R-phrases was evaluated more conservatively than the risk assessment method by OEL. And the risk assessment method by $\mathrm{H}$-statements was evaluated more conservatively than the risk assessment method by R-phrases. Conclusions: The CHARM model was gradually conservatively assessed as it proceeded in the next step without quantitative information for individual workplaces. The CHARM is expected to help identify the risk if the hazards and exposure levels of chemicals were identified in individual workplaces. For CMR substances, although CHARM is highly evaluated for hazards, the risk is assessed to be low if exposure levels are assessed low. When evaluating the risk of highly hazardous chemicals such as CMR substances, we believe the model should be adapted to be more conservative and classify these as higher risk. Int J Occup Med Environ Health 2018;31(4):491-501
\end{abstract}

Key words:

Exposure, Hazard, Risk assessment, Occupational, Work environment, Chemical hazard

Funding: this study was supported by the Korea Occupational Safety and Health Agency (KOSHA) and a research grant from Korea University (KU) (grant manager: Prof. Sang-Hoon Byeon, Ph.D).

Received: September 9, 2016. Accepted: June 12, 2017.

Corresponding author: Sang-Hoon Byeon, Korea University, Department of Health Science, Anam-ro 145, 136-701 Seongbuk-gu, Seoul, South Korea (e-mail: shbyeon@korea.ac.kr). 


\section{INTRODUCTION}

Many various chemicals are used in the industrial and technology sectors. In South Korea, chemicals are indispensable elements in industrialized modern society, and many kinds of chemicals are used in large quantities [1]. The use of so many chemicals has meant that occupational diseases associated with chemical exposure in South Korea increased from 373 cases in 2011 to 436 cases in 2012 [2]. Cases of acute poisoning and even death as a result of exposure to n-hexane, trichloroethylene, dimethylformamide have occurred as well as diseases caused by unclear chemicals case at the workplaces have been shown to workers [3].

In many countries, chemical risk management schemes have been developed. A series of chemical accidents occurred during the 1970's in the United States (US), and following growing concerns regarding chemical-induced cancer, in 1978 the US government developed the National Toxicology Program (NTP). In Europe, various methods used for assessing the potential risk and harm of chemicals have been consolidated to create REACH (Registration, Evaluation, Authorization and Restriction of Chemicals), a new legislation introduced in 2006 for managing chemicals [4]. Similarly to REACH, the South Korean government established the "Act on the Registration and Evaluation, etc. of Chemical Substances" [5] to manage chemical substances.

Chemical risk assessment needs to address the following area:

- establish what the potential effect of exposure to a chemical, at specified levels, would have on a worker's physical health;

- quantify toxicity information;

- establish standards and protocols for chemical management [6].

In 1998, the United Kingdom (UK) Health and Safety Executive (HSE) introduced a Control Banding (CB) method to determine the recommended level of risk for chemicals [7]. Control banding has been used globally as a chemical risk assessment method by the International Labor Organization (ILO) [8]. The HSE developed the Control of Substances Hazardous to Health $(\mathrm{COSHH})$ Essentials for the management of chemical substances in small and medium-sized enterprises [9]. The Control of Substances Hazardous to Health Essentials is a chemical substance management method for risk assessment and management that is based on the CB method [10].

In South Korea, a study of the methods used to introduce risk assessment was carried out in 2004. This study examined whether the methods were appropriate and essential, and how they should be introduced [11]. An additional study was conducted in 2009, which focused specifically on the introduction to risk assessment system [12]. In 2012, the Korea Occupational Safety and Health Agency (KOSHA) developed the Chemical Hazard Risk Management (CHARM) model, which was developed with reference to COSHH Essentials [13].

A recent study investigated advanced techniques for chemical risk assessment, including fire and explosion hazards [14], but there has not been much research on whether the CHARM model enables better management of chemicals. So, in this study, we have analyzed CHARM model of 59 kinds of carcinogenic, mutagenic or reprotoxic (CMR) substances in both managed working environment measurement and with usage information.

\section{MATERIAL AND METHODS}

Chemical Hazard Risk Management (CHARM) methods Chemical Hazard Risk Management is a risk assessment method developed in South Korea in order to better manage health risks posed to workers from workplace chemicals. The CHARM uses information on work environment measurement and daily usage and may use the physical state even if there is no work environment measurement information in individual workplaces. 
Table 1. Grading of chemical hazard level based on time-weighted average occupational exposure limits (TWA-OELs [18])*

\begin{tabular}{lcc}
\hline \multirow{2}{*}{$\begin{array}{c}\text { Hazard } \\
\text { level }\end{array}$} & \multicolumn{3}{c}{ TWA-OELs } \\
\cline { 2 - 3 } & dustiness (D) & volatility $(\mathrm{V})$ \\
\hline 1 & $1 \mathrm{mg} / \mathrm{m}^{3}<\mathrm{D} \leq 10 \mathrm{mg} / \mathrm{m}^{3}$ & $50 \mathrm{ppm}<\mathrm{V} \leq 500 \mathrm{ppm}$ \\
2 & $0.1 \mathrm{mg} / \mathrm{m}^{3}<\mathrm{D} \leq 1 \mathrm{mg} / \mathrm{m}^{3}$ & $5 \mathrm{ppm}<\mathrm{V} \leq 50 \mathrm{ppm}$ \\
3 & $0.01 \mathrm{mg} / \mathrm{m}^{3}<\mathrm{D} \leq 0.1 \mathrm{mg} / \mathrm{m}^{3}$ & $0.5 \mathrm{ppm}<\mathrm{V} \leq 5 \mathrm{ppm}$ \\
4 & $\mathrm{D}<0.01 \mathrm{mg} / \mathrm{m}^{3}$ & $\mathrm{~V}<0.5 \mathrm{ppm}$ \\
\hline
\end{tabular}

* Based on: Korea Occupational Safety and Health Agency. [Chemical risk assessment manual] [13].

Table 2. Grading of chemical hazard level based on Risk phrases (R-phrases)/Hazard statements (H-statements)*

\begin{tabular}{lll}
\hline $\begin{array}{c}\text { Hazard } \\
\text { level }\end{array}$ & \multicolumn{1}{c}{ R-phrases } & \multicolumn{1}{c}{ H-statements } \\
\hline 1 & $\begin{array}{l}\text { R36, R36/38, R38 and all substances that do not have } \\
\text { R-phrases in groups 2-4 }\end{array}$ & $\mathrm{H} 315, \mathrm{H} 319$ \\
2 & R20, R20/21, R20/21/22, R20/22, R21, R21/22, R22 & $\mathrm{H} 302, \mathrm{H} 312, \mathrm{H} 332$ \\
3 & R23, R23/24, R23/24/25, R24, R24/25, R25, R34, R35, R36/37, $\mathrm{H} 301, \mathrm{H} 311, \mathrm{H} 314, \mathrm{H} 315, \mathrm{H} 317, \mathrm{H} 318, \mathrm{H} 319, \mathrm{H} 330$, \\
& R36/37/38, R37, R37/38, R41, R43, R48/20, R48/20/21, & $\mathrm{H} 331, \mathrm{H} 335, \mathrm{H} 373$ \\
& $\begin{array}{l}\text { R48/20/21/22, R48/20/22, R48/21, R48/21/22, R48/22 } \\
\text { R26, R26/27, R26/27/28, R26/28, R27, R27/28, R28, }\end{array}$ & $\mathrm{H} 300, \mathrm{H} 310, \mathrm{H} 317, \mathrm{H} 330, \mathrm{H} 334, \mathrm{H} 351, \mathrm{H} 360, \mathrm{H} 361$, \\
& $\begin{array}{l}\text { Carc. cat. 3 R40, R48/23, R48/23/24, R48/23/24/25, } \\
\text { R48/23/25, R48/24, R48/24/25, R48/25, R60, R61, R62, R63, }\end{array}$ & \\
& Muta. cat. 3 R40, R42, R42/43, R45, R46, R49 & \\
\hline
\end{tabular}

* Based on: Korea Occupational Safety and Health Agency. [Chemical risk assessment manual] [13].

R20 - harmful by inhalation; R21 - harmful in contact with skin; R22 - harmful if swallowed; R23 - toxic by inhalation; R24 - toxic in contact with skin; R25 - toxic if swallowed; R26 - very toxic by inhalation; R27 - very toxic in contact with skin; R28 - very toxic if swallowed. R34 - causes burns; R35 - causes severe burns; R36 - irritating to eyes; R37 - irritating to respiratory system; R38 - irritating to skin. R40 - possible risks of irreversible effects; R41 - risk of serious damage to eyes; R42 - may cause sensitization by inhalation; R43 - may cause sensitization by skin contact; R45 - may cause cancer; R46 - may cause heritable genetic damage; R48 - danger of serious damage to health by prolonged exposure; R49 - may cause cancer by inhalation.

R60 - may impair fertility; R61 - may cause harm to the unborn child; R62 - possible risk of impaired fertility; R63 - possible risk of harm to the unborn child.

H300 - fatal if swallowed; $\mathrm{H} 301$ - toxic if swallowed; H302 - harmful if swallowed.

H310 - fatal in contact with skin; H311 - toxic in contact with skin; H312 - harmful in contact with skin; H314 - causes severe skin burns and eye damage; $\mathrm{H} 315$ - causes skin irritation; H317 - may cause an allergic skin reaction; H318 - causes serious eye damage; H319 - causes serious eye irritation. $\mathrm{H} 330$ - fatal if inhaled; H331 - toxic if inhaled; H332 - harmful if inhaled; H334 - may cause allergy or asthma symptoms of breathing difficulties if inhaled; $\mathrm{H} 335$ - may cause respiratory irritation.

H340 - may cause genetic defects, (state route of exposure if it is conclusively proven that no other routes of exposure cause the hazard); H341 - suspected of causing genetic defects (state route of exposure if it is conclusively proven that no other routes of exposure cause the hazard). H350 - may cause cancer (state route of exposure if it is conclusively proven that no other routes of exposure cause the hazard); H351 - suspected of causing cancer (state route of exposure if it is conclusively proven that no other routes of exposure cause the hazard).

H360 - may damage fertility or the unborn child (state specific effect if known) (state route of exposure if it is conclusively proven that no other routes of exposure cause the hazard); $\mathrm{H} 361$ - suspected of damaging fertility or the unborn child (state route of exposure if it is conclusively proven that no other routes of exposure cause the hazard).

H373 - may cause damage to organs through prolonged or repeated exposure (state all organs affected, if known) through prolonged or repeated exposure (state route of exposure if it is conclusively proven that no other routes of exposure cause the hazard).

Carc. - carcinogenic; cat. - category; muta. - mutagenic. 
Table 3. Grading of chemical exposure level based on work environment measurements*

\begin{tabular}{ll}
\hline Exposure level & Exposure level determination \\
\hline 1 & $10 \% \leq \frac{\text { work environment measurements }}{\text { TWA-OEL }} \times 100<50 \%$ \\
3 & $50 \% \leq \frac{\text { work environment measurements }}{\text { TWA-OELs }} \times 100 \leq 100 \%$ \\
4 & $100 \%<\frac{\text { work environment measurements }}{\text { TWA-OELs }} \times 100$ \\
\hline
\end{tabular}

* Based on: Korea Occupational Safety and Health Agency. [Chemical risk assessment manual] [13].

TWA-OELs - time weighted average-occupational exposure limits [18].

Table 4. Estimation of substance physical property and amount used for determination of exposure level*

\begin{tabular}{lccc}
\hline \multirow{2}{*}{ Substance amount } & \multicolumn{3}{c}{ Volatility and dustiness } \\
\cline { 2 - 4 } \multicolumn{1}{c}{ low } & medium & high \\
\hline $1 \mathrm{kl}(\mathrm{t})$ & 2 & $3\left(4^{* *}\right)$ & 4 \\
$11(\mathrm{~kg})$ to $1 \mathrm{kl}(\mathrm{t})$ & 2 & 3 & 3 \\
$<11(\mathrm{~kg})$ & 1 & 1 & 2 \\
\hline
\end{tabular}

* Based on: Korea Occupational Safety and Health Agency. [Chemical risk assessment manual] [13].

** Dustiness.

Table 5. Determination of the risk level by Chemical Hazard Risk Management (CHARM) model developed in South Korea*

\begin{tabular}{lccccccl}
\hline & \multicolumn{9}{c}{ Exposure level } & \multicolumn{3}{c}{ Risk } \\
\cline { 2 - 7 } & Hazard level & 2 & 3 & 4 & calculated & level & description \\
\hline 1 & 1 & 2 & 3 & 4 & 1,2 & 1 & low risk \\
2 & 2 & 4 & 6 & 8 & 3,4 & 2 & medium risk \\
3 & 3 & 6 & 9 & 12 & $6,8,9$ & 3 & high risk \\
4 & 4 & 8 & 12 & 16 & 12,16 & 4 & very high risk \\
\hline
\end{tabular}

* Based on: Korea Occupational Safety and Health Agency. [Chemical risk assessment manual] [13].

The CHARM determines the risk as an evaluation assessment of the hazard level and exposure level. The hazard level assessments for CHARM are generally carried out by applying the most appropriate of 4 methods which are respectively checking, whether they are relevant to CMR substance or not, chemical exposure standard, Risk phrase (R-phrase), or Globally Harmonized System (GHS) phrase (Hazard statement (H-statement)), according to chemical information. Initially, it is determined whether the chemical is a CMR substance. 
Table 6. Methods of Chemical Hazard Risk Management (CHARM) [13] model developed in South Korea

\begin{tabular}{llc}
\hline \multicolumn{1}{c}{ Exposure level determination } & Hazard level determination & Method No. \\
\hline $\begin{array}{l}\text { Work environment measurements } \\
\text { (substance concentration in work environment) }\end{array}$ & & \\
& CMR substance & 1 \\
& occupational exposure limits & 2 \\
& R-phrases & 3 \\
& H-statements & 4 \\
Non-work environment measurements & & \multicolumn{2}{|c}{} \\
(substance physical property and amount used) & CMR substance & 5 \\
& occupational exposure limits & 6 \\
& R-phrases & 7 \\
\hline
\end{tabular}

CMR - carcinogenic, mutagenic or reprotoxic; R-phrases - risk phrases; H-statements - hazard statements.

Table 7. Worker exposure information for chosen chemical substances in South Korea, 2009

\begin{tabular}{llccc}
\hline \multicolumn{1}{c}{ Substance } & CAS No. & $\begin{array}{c}\text { TWA-OEL } \\
{\left[\mathrm{mg} / \mathrm{m}^{3}\right]}\end{array}$ & $\begin{array}{c}\text { Substance concentration } \\
\text { in work environment } \\
{\left[\mathrm{mg} / \mathrm{m}^{3}\right]}\end{array}$ & $\begin{array}{c}\text { Usage for each } \\
\text { plant* } \\
{[\mathrm{t} / \mathrm{day}]}\end{array}$ \\
\hline Stoddard solvent & $8052-41-3$ & 525.00 & 2.47 & 8.44 \\
1,2,3-Trichloropropane & $96-18-4$ & 60.00 & 0.74 & $1.50 \times 10^{-3}$ \\
N,N-Dimethyl acetamide & $127-19-5$ & 35.00 & 0.61 & $2.53 \times 10^{2}$ \\
Ethylene dichloride & $107-06-2$ & 40.00 & 0.39 & $4.98 \times 10^{3}$ \\
Acrylonitrile & $107-13-1$ & 4.50 & 0.03 & 0.89 \\
2-Methoxyethyl acetate & $110-49-6$ & 24.00 & 0.09 & $3.30 \times 10^{-2}$ \\
1,2-Epoxypropane & $75-56-9$ & 5.00 & 0.05 & 0.06 \\
Hexane(n-hexane) & $110-54-3$ & 180.00 & 0.76 & $1.05 \times 10^{3}$ \\
1,4-Dioxane & $123-91-1$ & 72.00 & 2.95 & 8.40 \\
Dichloromethane & $75-09-2$ & 175.00 & 2.95 & 52.57 \\
Hydroquinone & $123-31-9$ & & 0.01 & $3.00 \times 10^{-5}$ \\
2-Butoxyethanol & $111-76-2$ & 97.00 & 1.29 & 7.22 \\
1,1,2-Trichloroethane & $79-00-5$ & 55.00 & 0.15 & 0.13 \\
Methyl n-butyl ketone & $591-78-6$ & 20.00 & 0.38 & 0.23 \\
Methoxyethanol & $109-86-4$ & & 0.23 & 0.02 \\
Vanadium pentoxide & $1314-62-1$ & 0.05 & 0.01 & 0.04 \\
\hline
\end{tabular}

CAS - Chemical Abstracts Service.

TWA-OEL - time-weighted average-occupational exposure limit [18]. 
The Table 1 and 2 present the estimation for hazard level for non-CMR substance. Grading of the Table 1 is based on exposure limit. Grading of the Table 2 is based on R-phrase/H-statement. The Table 3 and 4 present estimation for exposure level. Grading of the Table 3 is based on work environment measurements. Grading of the Table 4 is based on physical state and amount. The Table 5 was a criterion for determining the risk. For CMR substances, the hazard level is assigned grade 4. If so, then the hazard level should be assessed according to the exposure limit in the Table 1. If there is no exposure limit for the chemical, R-phrases should be used for the risk assessment. Finally, if there are no R-phrases, then H-statements should be used. Then the hazard level may be assessed according to R-phrase and $\mathrm{H}$-statements in the Table 2.

Exposure level according to CHARM is classified in 2 ways depending on the presence or absence of the working environment measurement results. If the working environment measurement results exist, these may be used for taking advantage of measurement results. If they do not exist, then the handling volume and the physical properties (e.g., fugacity and volatility) of the substance are considered. When the working environment measurement results do exist, exposure level is evaluated according to the criteria of the Table 3 , the results are divided by the working environment measurement to give time-weighted average-occupational exposure limits (TWA-OELs) of a chemical. When the working environment measurement results do not exist, exposure level is evaluated according to the criteria of the Table 4 for the handling volume and the physical properties (e.g., fugacity and volatility) of the chemicals. After the evaluation of hazard and exposure levels of chemicals, the risk level is determined by a combination of the exposure level and the hazard level as in the Table 5 [13]. The hazard and exposure grading $(1,2,3,4)$ is merely the classification to classify low and high of evaluation.
Therefore the definition of the meaning of hazard and exposure grading corresponding to hazard and exposure grading is more important.

The Table 6 shows a representation about the method of CHARM. Method 1 to 4 assessed exposure levels using the working environment measurement results, and method 5 to 8 assessed exposure levels using the handling volume and the physical properties of the chemical substances.

\section{Chemical substances assessed in the study}

In South Korea, in accordance with the "Occupational Safety and Health Act" [15], measurements of the working environment are conducted twice a year. And workplace survey is also conducted every 5 years.

Hazardous chemicals that have been assessed by working environment measurements include 113 organics compounds, 23 metals, 17 acids and alkalis, and 15 gas-state substances. These are harmful chemicals that require management according to the "Local Rules on Occupational Safety and Health Standards" [16].

In this study, we used 59 CMR substances. Fifty-nine CMR substances, which require management, are included in the actual working environment data released by the Ministry of Employment and Labor (MOEL) and KOSHA [17]. These have working environment measurements and usage information which were used in assessments. The Table 7 shows data for 16 out of the 59 subject substances. These substances include stoddard solvent, acrylonitrile, and dichloromethane. The daily usage is the average daily usage for each plant.

\section{RESULTS}

Fifty-nine CMR substances, the measured values of which may be obtained from the actual working environment measurement data, were subjected to several risk assessment methods outlined in the CHARM model [13]. Information about the substances was obtained from the 
Table 8. Hazard and exposure levels for chosen chemical substances in South Korea, 2009

\begin{tabular}{llllllcc}
\hline \multirow{2}{*}{\multicolumn{1}{c}{ Substance }} & & \multicolumn{5}{c}{ Hazard level } & \multicolumn{3}{c}{ Exposure level } \\
\cline { 3 - 8 } & CAS No. & CMR & OELs & R-phrases & H-statements & $\begin{array}{c}\text { substance } \\
\text { concentration } \\
\text { in work environment }\end{array}$ & $\begin{array}{c}\text { substance physical } \\
\text { property and amount }\end{array}$ \\
\hline Stoddard solvent & $8052-41-3$ & 4 & 1 & 4 & 4 & 1 & 2 \\
1,2,3-Trichloropropane & $96-18-4$ & 4 & 2 & 4 & 4 & 1 & 2 \\
N,N-Dimethylacetamide & $127-19-5$ & 4 & 2 & 4 & 4 & 1 & 2 \\
Ethylene dichloride & $107-06-2$ & 4 & 2 & 4 & 4 & 1 & 3 \\
Acrylonitrile & $107-13-1$ & 4 & 3 & 4 & 4 & 1 & 3 \\
2-Methoxyethyl acetate & $110-49-6$ & 4 & 2 & 4 & 4 & 1 & 3 \\
1,2-Epoxypropane & $75-56-9$ & 4 & 3 & 4 & 4 & 1 & 3 \\
Hexane (n-hexane) & $110-54-3$ & 4 & 1 & 4 & 4 & 1 & 3 \\
1,4-Dioxane & $123-91-1$ & 4 & 2 & 4 & 4 & 1 & 4 \\
Dichloromethane & $75-09-2$ & 4 & 1 & 4 & 4 & 1 & 2 \\
2-Butoxyethanol & $111-76-2$ & 4 & 2 & 2 & 4 & 1 & 3 \\
1,1,2-Trichloroethane & $79-00-5$ & 4 & 2 & 4 & 4 & 1 & 3 \\
Methyl n-butyl ketone & $591-78-6$ & 4 & 2 & 4 & 4 & 1 & 2 \\
Diethanolamine & $111-42-2$ & 4 & 4 & 3 & 4 & 1 & 1 \\
p-Nitrochlorobenzene & $100-00-5$ & 4 & 4 & 4 & 4 & 1 & 3 \\
Vanadium pentoxide & $1314-62-1$ & 4 & 3 & 4 & 4 & 2 & 3 \\
\hline
\end{tabular}

OELs - occupational exposure limits.

Other abbreviations as in Tables 1, 2, 5 and 6.

Globally Harmonized System - Material Safety Data Sheets (GHS-MSDS) which are released by KOSHA via the internet [18].

The Table 8 shows the results of the hazard and exposure level for 16 out of the 59 CMR substances. The Figure 1 and 2 show the results of evaluating the risk by combining the results of the evaluation of the hazard and exposure level of the target substance. The Figure 1 shows the results for the CMR substances when exposure was assessed using the working environment measurements method. And the Figure 2 shows the results of the method for evaluating the exposure level with a combination of the handling volume and physical properties (e.g., fugacity and volatility) of chemical substances when working environment measurements as exposure were not used.
The comparison of the Figure 1 and 2 shows the risks evaluated as higher when working environment measurements were not applied. The hazard was evaluated most conservatively in method 1 , which resulted in assignment of grade 4 (due to CMR substances), and in method 4 where H-statement information was used. In method 1 to 4 , application of the hazard assessment was gradually more conservatively applied as the risk assessment process moved to the next phase. The results of method 1 and 4 were the same. Since the hazard level of CMR substances was evaluated equal to grade 4. Method 3, in which the hazard grade was determined using R-phrases, appears more conservative than method 2, which used the exposure level, and method 4, where the hazard grade was determined using the H-statements, appeared more conservative than method 3 . 

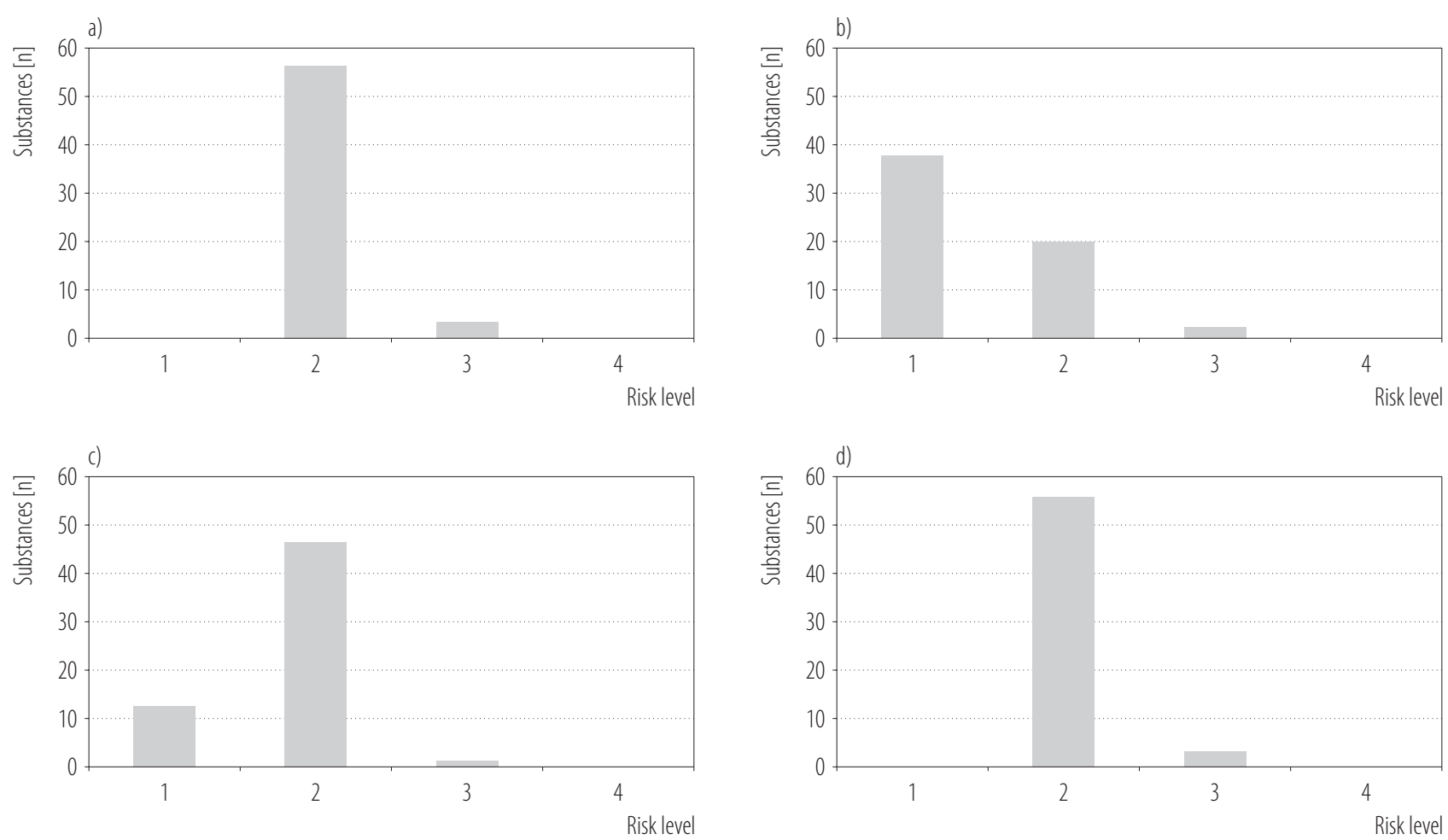

Risk level: 1 - lowest risk, 4 - highest risk.

Fig. 1. Chemical Hazard Risk Management (CHARM) [13] assessments using the work environment measurements: a) method 1 - CMR substance, b) method 2 - OELs, c) method 3 - R-phrases, d) method 4 - H-statements

The methods, where working environment measurements were used, evaluated health risks as lower than the method using the fugacity and physical properties of the chemical under assessment. Method 5, where the chemical was a CMR substance, and method 8 , in which $\mathrm{H}$-statements were used, produced identical assessment results and were conservatively evaluated. The results of method 5 and 8 were the same. Since the hazard level of CMR substances was evaluated equal to grade 4. In method 6 to 8, application of the hazard assessment was gradually conservatively applied as the assessment proceeded to the next phase.

The CHARM model uses R-phrase information before using $\mathrm{H}$-statements, which are only used if there is no R-phrase information [13]. For method 3, 4, 7, and 8 in Figure 1 and 2, the result of hazard assess- ment appeared differently as a result of using R-phrase and $\mathrm{H}$-statement.

\section{DISCUSSION}

In the CHARM assessment model, the standards used for assessing whether to apply the R-phrase and H-statements, or whether to apply the physical properties method are identical to the ones used by the Control Banding (CB) model [19,20]. Furthermore, CHARM, like CB, indicates risk level by combining the harmfulness grade and exposure level grade [21].

The results for the evaluation of $59 \mathrm{CMR}$ substances showed that the health risks were assessed to be lower when using the working environment measurement results compared to the other methods. This is because the working environment measurements in the actual workplace 

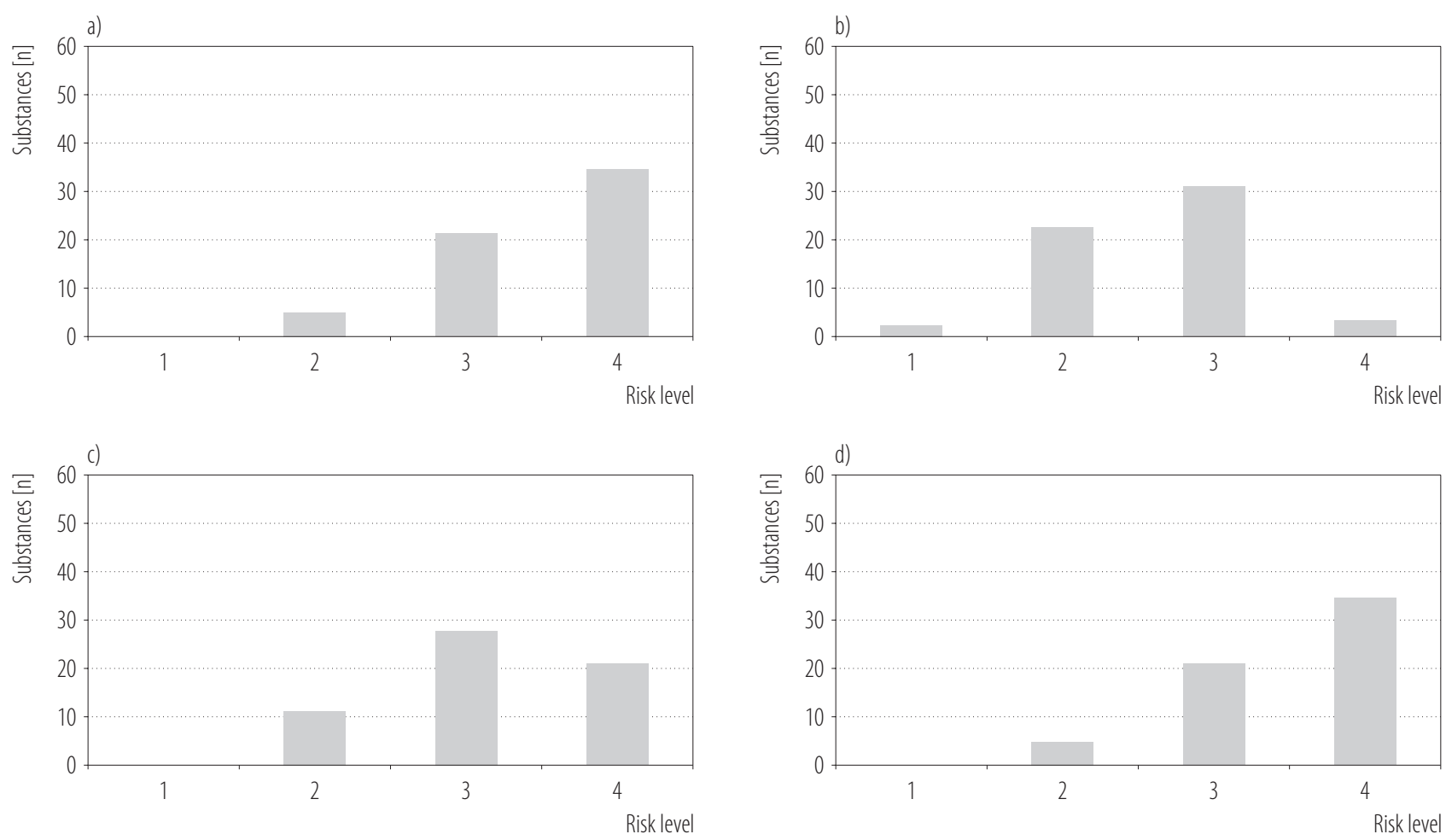

Risk level: 1 - lowest risk, 4 - highest risk.

Fig. 2. Chemical Hazard Risk Management (CHARM) [13] assessments when work environment measurements are not available: a) method 5 - CMR substance, b) method 6 - OELs, c) method 7 - R-phrases, d) method 8 - H-statements

assessment were found to be lower than the occupational exposure limit. That is, even when assessing highly hazardous CMR substances, if the exposure level is low, then the risk will be assessed as low. In the harmfulness assessment, risks using the R-phrase and H-statement were assessed to be higher than when the risk was assessed using the occupational exposure limit. Accordingly, the CHARM model assessed the risk as high when no quantitative information (such as working environment measurements or handling volume) was available. Therefore, the assessment of chemical risk by CHARM improves safety by conservatively assessing the risk level, particularly if there is little available information on the chemical. And if all 4 types of data are available, Method 2 is considered to be most effective because this is a method of using actual quantitative information of the workplace.
Hazardous chemical information used in this study consisted of R-phrases, H-statements, occupational exposure limits, the physical and chemical properties (fugacity/volatility). The risk assessment results obtained by applying the R-phrases and H-statements were evaluated differently because R-phrases and H-statements of some substances in the MSDSs provided by KOSHA were classified into different levels. For example, nitromethane was classified as acutely toxic (R22) for R-phrase although it was classified not only as acutely toxic (H302) but also carcinogenic for the H-statement (H351) [18]. In Europe, there are also various classification levels according to CHIP Regulations [22] and CLP Regulation [23] in conjunction with the hazard identification of chemicals [24]. The Korean MSDS is compiled by the Standard for classification and labeling of chemical substance and material 
safety data sheet [25]. In addition, following the enforcement of the Globally Harmonized System (GHS) [26], the classification display and MSDS are being newly prepared, alongside a study for its effective development and application [27]. However, the classification levels for the hazards and risks posed by chemical substances have not been applied in the same way. Therefore, additional studies are required to improve the system and ensure that more accurate chemical information may be delivered by clear classification standards.

\section{CONCLUSIONS}

Health risks posed by CMR substances assessed without working environment measurement results and occupational exposure limits are judged as higher than when the working environment measurement results and occupational exposure limits are available. Moreover, if the exposure level is assessed as low, even though the hazard level is assessed as high, then the overall risk is assessed as low. The CHARM model was developed for chemical management in individual workplaces. The CHARM is expected to help identify the risk if the hazards and exposure levels of chemicals were identified in individual workplaces. However, risks posed by highly hazardous chemicals such as CMR substances, tend to be assessed as low using the CHARM model. When evaluating the risk of highly hazardous chemicals such as CMR substances, we believe the model should be adapted to be more conservative and classify these as higher risk.

\section{REFERENCES}

1. Park JK, Seo YW, Kan SY. [A study on the improvement of the chemical accident response system]. Seoul: Korea Environment Institute; 2013. p. 1-56. Korean.

2. Korea Ministry of Employment and Labor. [Implementation results of the medical examination of the workers (2012)]. Sejong: Ministry of Employment and Labor; 2014. p. 9-17. Korean.
3. Byeon SH, Choi HC, Park HJ, Lee CM, Lee SK, Lee SK, et al. [Risk assessment of hazardous chemical substances in 2012(I)]. Incheon: Occupational Safety and Health Research Institute; 2012. p. 1-6. Korean.

4. Lim $\mathrm{CH}$. [Limits expected of hazards and risk assessment of industrial chemicals]. Vol. 280. Incheon: Korea Occupational Safety and Health Agency; 2012. p. 14-19. Korean.

5. Act on the Registration and Evaluation, etc. of Chemical Substances - "K-REACH”, Act No. 11789 (May 22, 2013).

6. Kim CN, No JH, Won JG, No YM, Kim KY, Yang JY, et al. Risk assessment of toxic chemicals. Incheon: Occupational Safety and Health Research Institute; 2011. p. 1-11. Korean.

7. Zalk DM, Nelson DI. History and evolution of control banding: A review. J Occup Environ Hyg. 2008;5(5):330-46, https://doi.org/10.1080/15459620801997916.

8. Hashimoto H, Goto T, Nakachi N, Suzuki H, Takebayashi T, Kajiki S, et al. Evaluation of the control banding method: Comparison with measurement-based comprehensive risk assessment. J Occup Health. 2007;49:482-92, https://doi. org/10.1539/joh.49.482.

9. Hay A. Controlling exposure to chemicals: A simple guide. Ann N Y Acad Sci. 2006;1076:790-9, https://doi.org/10.1196/ annals.1371.016.

10. Fingerhut M. Global qualitative risk management (control banding) activities. Ind Health. 2008;46:305-7, https://doi. org/10.2486/indhealth.46.305.

11. Park DY, Jae MS, Yun JK, Park JS, Park JH, Park D. [Study on paradigm shift of the national OSH institutions based on risk assessment for general industry]. Seoul: Ministry of Employment and Labor; 2004. p. 1-94. Korean.

12. Park DY, Baek DM, Lee YS, Kim SK, Park JS, Kim HK, et al. [Development and introduction of an OSH regulatory framework based on risk assessment for Korean OSH regulation systems and policy]. Incheon: Occupational Safety and Health Research Institute; 2009. p. 1-175. Korean.

13. Korea Occupational Safety and Health Agency. [Chemical risk assessment manual]. Incheon: Korea Occupational Safety and Health Agency; 2012. p. 1-95. Korean. 
14. Yun JD, Kim JH, Jeong YH, Yun JY. [A study on the research in risk assessment methods]. Daejeon: Occupational Safety and Health Research Institute; 2013. p. 1-188. Korean.

15. Occupational Safety and Health Act, Act No. 11882 (Jun 4, 2013).

16. The Ministry of Government Legislation. [Local rules on occupational safety and health standard, Ordinance of the Ministry of Employment and Labor, No. 77 (Mar 21, 2013)]. Korean.

17. Korea Occupational Safety and Health Agency. [Refund status reports national industrial work environment 2009]. Seoul: Ministry of Employment and Labor; 2010. Korean.

18. Msds.kosha.or.kr [Internet]. Incheon: Korea Occupational Safety and Health Agency; Globally harmonized system Material safety data sheet (GHS-MSDS) [cited 2014 Jan 11]. Available from: http://msds.kosha.or.kr.

19. Brooke IM. A UK scheme to help small firms control health risks from chemicals: Toxicological considerations. Ann Occup Hyg. 1998;42(6):377-90.

20. Maidment SC. Occupational hygiene considerations in the development of a structured approach to select chemical control strategies. Ann Occup Hyg. 1998;42(6):391-400.

21. Russell RM, Maidment SC, Brooke IM, Topping MD. An introduction to a UK scheme to help small firms control health risks from chemicals. Ann Occup Hyg. 1998;42(6):367-76.
22. Legislation.gov.uk [Internet]. United Kingdom the National Archives: The chemicals (hazard information and packaging for supply) regulations 2009 [cited 2014 Sep 20]. Available from: http://www.legislation.gov.uk/uksi/2009/716/contents/ made.

23. Regulation (EC) No 1272/2008 of the European Parliament and of the Council of 16 December 2008 on classification, labelling and packaging of substances and mixtures, amending and repealing Directives 67/548/EEC and 1999/45/EC, and amending Regulation (EC) No 1907/2006. Off J Eur Union L 353, p. 1-1355 (Dec 31, 2008).

24. Garrod ANI, Evans PG, Davy CW. Risk management measures for chemicals: The "COSHH essentials" approach. J Exposure Sci Environ Epidemiol. 2007;17:48-54, https:// doi.org/10.1038/sj.jes.7500585.

25. Safe Chemicals. Standard for classification and labeling of chemical substance and material safety data sheet, Public notice No. 2013-37 (Aug 14, 2013).

26. United Nations. Globally harmonized system of classification and labelling of chemicals (GHS). 5th rev. New York and Geneva: United Nations; 2013. p. 1-536.

27. Lee SW, Oh SY, Kim TG. Overview of GHS (globally harmonized system) in Korea and the direction of further development. J Loss Prev Process Ind. 2013;26:904-7, https:// doi.org/10.1016/j.jlp.2012.12.001.

This work is available in Open Access model and licensed under a Creative Commons Attribution-NonCommercial 3.0 Poland License - http://creativecommons.org/ licenses/by-nc/3.0/pl/deed.en. 